\title{
Dynamic stabilization using the Dynesys system versus posterior lumbar interbody fusion for the treatment of degenerative lumbar spinal disease: a clinical and radiological outcomes-based meta-analysis
}

\author{
Chang-Hyun Lee, MD,' Tae-Ahn Jahng, MD, PhD, 2,3 Seung-Jae Hyun, MD, PhD, ${ }^{2}$ \\ Chi Heon Kim, MD, ${ }^{3-6}$ Sung-Bae Park, MD, ${ }^{7}$ Ki-Jeong Kim, MD, PhD, ${ }^{2}$ \\ Chun Kee Chung, MD, PhD, ${ }^{3-6}$ Hyun-Jib Kim, MD, PhD, ${ }^{2}$ and Soo-Eon Lee, MD ${ }^{2}$

\begin{abstract}
'Department of Neurosurgery, Ilsan Paik Hospital, Inje University College of Medicine, Goyang; '2Department of Neurosurgery, Spine Center, Seoul National University Bundang Hospital, Seongnam; ${ }^{3}$ Seoul National University College of Medicine, Seoul; ${ }^{4}$ Department of Neurosurgery and ${ }^{5}$ Clinical Research Institute, Seoul National University Hospital, Seoul; ${ }^{6}$ Neuroscience Research Institute, Seoul National University Medical Research Center, Seoul; and 'Department of Neurosurgery, SMG-SNU Boramae Medical Center, Seoul, Republic of Korea
\end{abstract}

OBJECTIVE The Dynesys, a pedicle-based dynamic stabilization (PDS) system, was introduced to overcome the drawbacks of fusion procedures. Nevertheless, the theoretical advantages of PDS over fusion have not been clearly confirmed. The aim of this study was to compare clinical and radiological outcomes of patients who underwent PDS using the Dynesys system with those who underwent posterior lumbar interbody fusion (PLIF).

METHODS The authors searched PubMed, Embase, Web of Science, and the Cochrane Database. Studies that reported outcomes of patients who underwent PDS or PLIF for the treatment of degenerative lumbar spinal disease were included. The primary efficacy end points were perioperative outcomes. The secondary efficacy end points were changes in the Oswestry Disability Index (ODI) and back and leg pain visual analog scale (VAS) scores and in range of motion (ROM) at the treated and adjacent segments. A meta-analysis was performed to calculate weighted mean differences (WMDs), 95\% confidence intervals, $Q$ statistics, and $I^{2}$ values. Forest plots were constructed for each analysis group.

RESULTS Of the 274 retrieved articles, 7 (which involved 506 participants [Dynesys, 250; PLIF, 256]) met the inclusion criteria. The Dynesys group showed a competitive advantage in mean surgery duration $(20.73$ minutes, $95 \% \mathrm{Cl} 8.76$ 32.70 minutes), blood loss $(81.87 \mathrm{ml}, 95 \% \mathrm{Cl} 45.11-118.63 \mathrm{ml})$, and length of hospital stay $(1.32$ days, $95 \% \mathrm{Cl} 0.23-2.41$ days). Both the Dynesys and PLIF groups experienced improved ODI and VAS scores after 2 years of follow-up. Regarding the ODI and VAS scores, no statistically significant difference was noted according to surgical procedure (ODI: WMD $0.12,95 \% \mathrm{Cl}-3.48$ to 3.72 ; back pain VAS score: WMD $-0.15 ; 95 \% \mathrm{Cl}-0.56$ to 0.26 ; leg pain VAS score: WMD -0.07 ; $95 \% \mathrm{Cl}-0.47$ to 0.32 ). The mean ROM at the adjacent segment increased in both groups, and there was no substantial difference between them (WMD 1.13; $95 \% \mathrm{Cl}-0.33$ to 2.59). Although the United States is the biggest market for Dynesys, no eligible study from the United States was found, and 4 of 8 enrolled studies were performed in China. The results must be interpreted with caution because of publication bias. During Dynesys implantation, surgeons have to decide the length of the spacer and cord pretension. These values are debatable and can vary according to the surgeon's experience and the patient's condition. Differences between the surgical procedures were not considered in this study.

CONCLUSIONS Fusion still remains the method of choice for advanced degeneration and gross instability. However, spinal degenerative disease with or without Grade I spondylolisthesis, particularly in patients who require a quicker recovery, will likely constitute the main indication for PDS using the Dynesys system.

http://thejns.org/doi/abs/10.3171/2015.10.FOCUS15426

KEY WORDS Dynesys; dynamic stabilization; fusion; lumbar; pedicle screw; meta-analysis

ABBREVIATIONS ASD = adjacent-segment degeneration; EBL = estimated blood loss; ODI = Oswestry Disability Index; PDS = pedicle-based dynamic stabilization; PLIF = posterior lumbar interbody fusion; ROM = range of motion; VAS = visual analog scale; WMD = weighted mean difference.

SUBMITTED August 27, 2015. ACCEPTED October 23, 2015.

INCLUDE WHEN CITING DOI: 10.3171/2015.10.FOCUS15426. 
$\mathrm{S}$ PINAL fusion has been the cornerstone of surgical treatment for degenerative spinal disease during the last 3 decades.$^{30}$ Fusion surgery has been associated with several undesirable effects, such as pseudarthrosis, nonunion, instrumentation failure, and adjacent-segment degeneration (ASD). ${ }^{18}$ Even if solid fusion occurs, persistent back pain sometimes haunts surgeons and patients. ${ }^{30}$ The Dynesys system (Zimmer, Inc.), a semirigid pediclebased dynamic stabilization (PDS) system, was introduced in an attempt to overcome the drawbacks of fusion procedures. ${ }^{11}$ Many in vitro and biomechanical studies have shown that this system can restrain the amount of flexibility through polyethylene-terephthalate cords and polycarbonate urethane spacers. ${ }^{38}$ In theory, it allows for less loading on the adjacent discs and facet joints and preserves motion of the treated segment..$^{5}$ Nevertheless, the theoretical advantages of nonfusion PDS over lumbar fusion, such as the prevention of ASD, have not been clearly confirmed or defined. ${ }^{21}$

Some clinical studies have found positive outcomes with improved Oswestry Disability Index (ODI) and visual analog scale (VAS) pain scores, as well as shorter recovery times, for patients with degenerative lumbar disease treated using the Dynesys system compared with those of patients who underwent fusion. ${ }^{18,25,27,33,38,39}$ Although the early outcomes have been promising, the long-term effects are still debated. ${ }^{11}$ Moreover, many recently published studies have reported contradictory results indicating that Dynesys may not provide a significant advantage for outcomes (clinical measurements, motion preservation, and adjacent-disc protection). ${ }^{4,7,10,35,38}$

Thus, we aimed to directly compare the radioglogical and clinical outcomes of patients who underwent PDS with the Dynesys system with those who underwent posterior lumbar interbody fusion (PLIF) for degenerative lumbar spinal disease by systemically reviewing all pertinent studies and meta-analyses.

\section{Methods}

\section{Search Strategy and Selection Criteria}

We performed a meta-analysis of clinical studies concerning the efficacy of Dynesys in accordance with Preferred Reporting Items for Systematic Reviews and MetaAnalyses (PRISMA) guidelines. We searched PubMed, Embase, Web of Science, and the Cochrane Database from inception to June 19, 2015, using the terms "Dynesys" or "semi-rigid" or "dynamic" together with "fusion." In addition, the reference lists of reviews of Dynesys were screened for qualifying studies. To qualify for inclusion, a study had to have compared clinical and radiological outcomes directly and differed only in surgical methods. The surgical methods were PLIF and Dynesys system-based PDS. Studies of individuals who underwent procedures that used other instruments (e.g., N-Flex, GRAF, an interspinous device, and/or Isobar) were not eligible. Biomechanical or single-treatment-arm studies also were not eligible. In the case of overlapping study populations, we used the most recent publication. There were no language restrictions on study eligibility.

\section{Statistical Analysis}

The primary efficacy end points were perioperative outcomes (surgery duration, estimated blood loss [EBL], and length of hospital stay). The secondary efficacy end points were changes in ODI and back and leg pain VAS scores and in range of motion (ROM) at the treated and adjacent segments after more than 2 years of follow-up. For the pooled effects, weighted mean differences (WMDs) and 95\% confidence intervals were calculated for continuous variables according to the consistency of measurement units. Random-effects or fixed-effects models were used depending on the heterogeneity of the studies included. To assess heterogeneity in the results of individual studies, we used the Cochran Q test and the Higgins $\mathrm{I}^{2}$ statistic $\left(\mathrm{I}^{2}>\right.$ $50 \%$ was used as a threshold to indicate significant heterogeneity). We assessed publication bias by visual inspection of funnel plots and by calculation of the $p$ values (1-sided) for Egger's intercept. All tests were 2-sided, and any $p$ value less than 0.05 was deemed significant. All statistical tests were performed using R 3.2.0 software (The R Foundation for Statistical Computing) and Review Manager 5.3 (The Cochrane Collaboration).

\section{Results}

\section{Search Results for Relevant Studies}

An initial search using subject headings identified 107 studies in PubMed, 155 studies in Embase, 10 studies in Web of Science, and 2 studies in the Cochrane Central Register of Controlled Trials. Among these 274 studies, 97 duplicated articles were excluded. Among the 177 remaining studies, 39 papers were case reports, review articles, letters, technical notes, or patents; therefore, these papers were excluded after screening. Twelve experimental studies and 14 studies concerning the cervical spine were also excluded. The remaining 112 studies were subjected to a full-text review. The reasons for excluding the remaining articles were as follows: the intervention did not use the Dynesys system $(\mathrm{n}=59)$; the intervention was for scoliosis $(\mathrm{n}=7)$; there was no comparative study or the study was compared with other fusion techniques (not PLIF) (n $=18$ ); or the scoring tools were incompatible with those identified in our study $(n=21)$. Finally, a total of 7 studies were included in the meta-analysis. The detailed selection process is shown in Fig. 1.

Table 1 shows general information regarding the 7 studies included in this analysis. The studies recruited 506 participants (250 patients in the Dynesys group, 256 patients in the PLIF group). Four studies were retrospective intervention-comparison studies, and 3 were prospective randomized studies. ${ }^{39}$ The average age of the 506 patients was 50.3 years (Dynesys group 49.0 years; PLIF group 51.6 years), and the proportions that were male were $50.8 \%$ and $48.0 \%$, respectively. The countries in which the studies were conducted included China $(n=4)$, the United King$\operatorname{dom}(n=1)$, Turkey $(n=1)$, and Taiwan $(n=1)$. There were no significant differences in ODI or VAS scores or in ROM between the Dynesys and PLIF groups at the baseline state.

\section{Risk of Bias of Included Studies}

Although the United States is the biggest market for the 


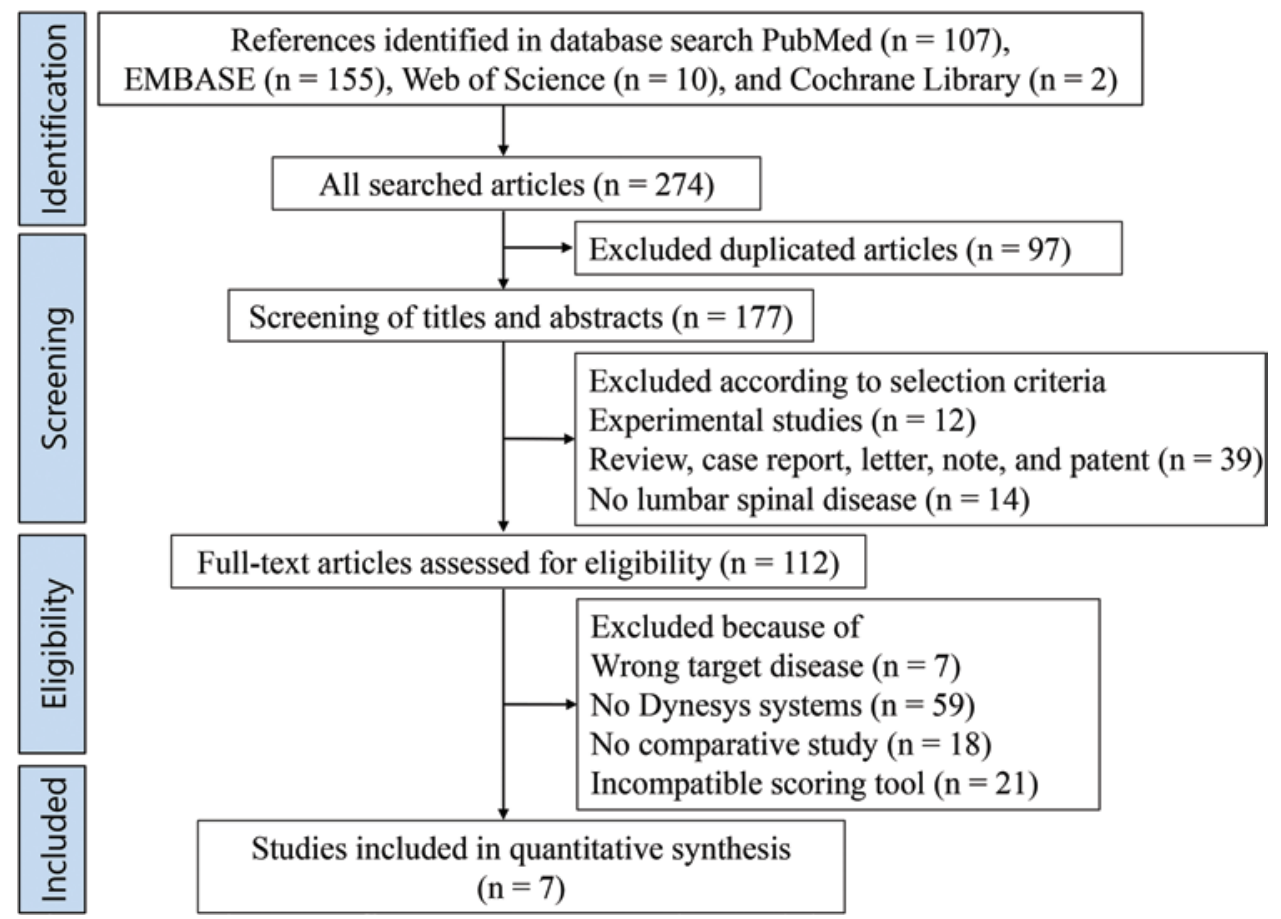

FIG. 1. Flow chart of identification of relevant studies.

Dynesys system, no eligible study from the United States was found. The Dynesys system is approved by the FDA only as a fusion device and not a motion-preservation device. Therefore, use of the Dynesys system as a PDS in the United States would be off-label, which may be a reason for there to be no publications from this country.

All the included studies were prospective or retrospective cohort studies of relatively high quality. The study by $\mathrm{Yu}$ et al. ${ }^{39}$ was a prospective trial. This study was single blind, and 3 participants dropped out. The authors described outcomes using change percentages. We changed the values to mean differences and standard deviations by combining the effect-size estimates., 3,22,23 Haddad et al. ${ }^{11}$ described "posterior conventional fusion" as a surgical method for the control group. Despite uncertainty about the surgical technique, the study was included to compare clinical outcomes at the final follow-up. The patients of 5 studies underwent single-level PLIF or PDS. 4,13,36,37,39 Two studies were concerned with surgery on 3 or fewer segments. ${ }^{9,38}$ Among the 7 studies, Kaner et al. ${ }^{13}$ included patients with Grade I or II spondylolisthesis, and the others included patients with only Grade I spondylolisthesis.

\section{Results of Individual Studies and Synthesis of Results}

The Dynesys group experienced better perioperative outcomes than the PLIF group, as shown in Fig. 2. The mean surgery time of the Dynesys group was 20.73 minutes (95\% CI 8.76-32.70 minutes) shorter than that of the PLIF group $\left(\mathrm{p}<0.01, \mathrm{I}^{2}=92 \%\right)$. The mean EBL was 81.87 $\mathrm{ml}$ (95\% CI 45.11-118.63 ml) less for the Dynesys group than for the PLIF group $\left(\mathrm{p}<0.01, \mathrm{I}^{2}=95 \%\right)$. The mean length of hospital stay of the Dynesys group was 1.32 days (95\% CI 0.23-2.41 days) shorter than that of the PLIF group $\left(\mathrm{p}=0.10, \mathrm{I}^{2}=57 \%\right)$.

Clinical and functional outcomes were assessed by ODI and back and leg pain VAS scores at baseline and after approximately 2 years of follow-up. There were no significant differences in the baseline ODI or VAS scores between the 2 groups. The ODI scores decreased to 37.81 and 37.69 in the Dynesys and PLIF groups, respectively. In the random-effects meta-analysis of 6 studies, no significant difference in the ODI scores was observed between the 2 surgical methods, as shown in Fig. 3 (WMD $0.12,95 \% \mathrm{CI}-3.48$ to $3.72, \mathrm{p}=0.95, \mathrm{I}^{2}=82 \%$ ). Regarding the back and leg pain VAS scores, no statistically significant differences were noted in the improvement of back and leg pain based on surgical procedure, although there was a trend toward better outcomes in the PLIF group, as shown in Fig. 2 (back pain VAS score: WMD -0.15, 95\% CI -0.56 to $0.26, p=0.47, I^{2}=62 \%$; leg pain VAS score: WMD $-0.07,95 \% \mathrm{CI}-0.47$ to $0.32, \mathrm{p}=0.71, \mathrm{I}^{2}=59 \%$ ).

The mean ROM at the treated segment changed from $6.64^{\circ}$ to $3.64^{\circ}$ ( $45.2 \%$ reduction) in the Dynesys group and from $6.73^{\circ}$ to $0.71^{\circ}$ (89.4\% reduction) in the PLIF group after approximately 2 years of follow-up. Although both groups showed substantially decreased ROM at the treated segment, the ROM of the PLIF group decreased significantly compared with that of the Dynesys group, as shown in Fig. 4 (WMD $-3.43^{\circ}$, 95\% CI -5.25 to -1.60 , $\left.\mathrm{p}<0.0002, \mathrm{I}^{2}=97 \%\right)$. A ROM increase at the adjacent segment indicates hypermobility and an increased risk of ASD. The ROM at the adjacent segment in the Dynesys and PLIF groups increased by $0.33^{\circ}$ and $1.15^{\circ}$, respectively (WMD $1.13^{\circ}, 95 \% \mathrm{CI}-0.33$ to $2.59, \mathrm{p}=0.13, \mathrm{I}^{2}=$ 


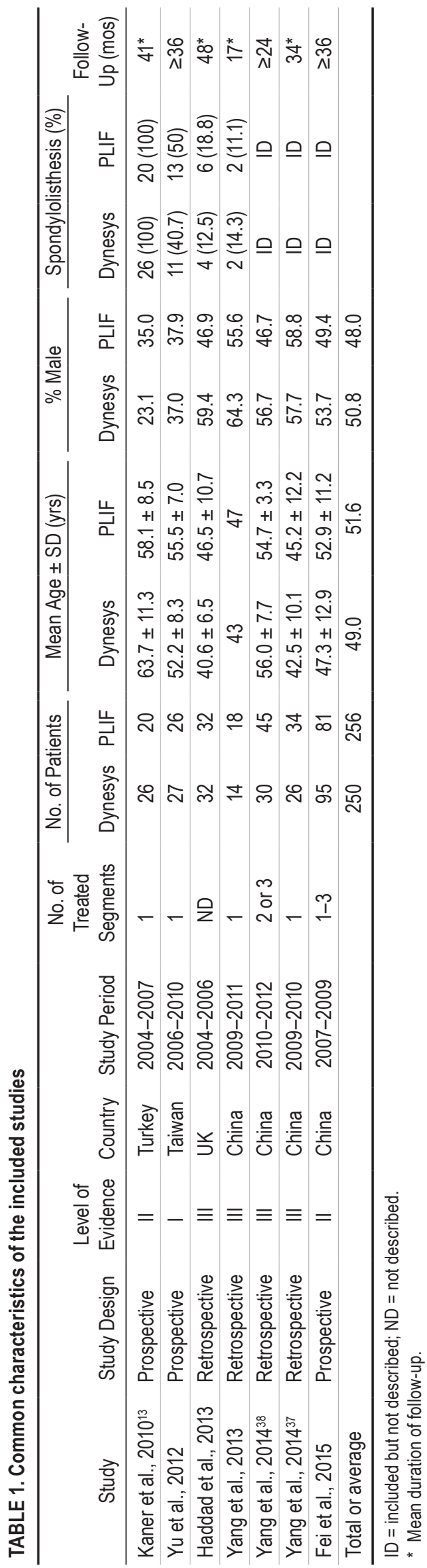

89\%). There were no significant differences between the 2 groups, as shown in Fig. 4.

Complications were reported in 6 papers. ${ }^{9,11,13,37-39} \mathrm{Ra}-$ diographically viewed screw loosening was observed in $6(2.54 \%)$ patients in the Dynesys group and $5(2.10 \%)$ patients in the PLIF group. One $(0.42 \%)$ patient in the Dynesys group and $3(1.26 \%)$ patients in the PLIF group experienced symptomatic screw loosening and underwent revision surgery. Revision surgery at the early postoperative state was needed in $3(1.27 \%)$ patients in the Dynesys group and 4 (2.10\%) patients in the PLIF group because of screw malposition or other hardware complications. Other complications, such as wound infection, dural tear, and failed-back surgery syndrome, were trivial.

\section{Publication Bias}

Funnel plots were constructed for changes in ODI and VAS scores, ROM, EBL, length of hospital stay, and surgery duration (shown in Fig. 5). The funnel plot shows asymmetry in ROM, which may indicate an underpowered analysis. The funnel plots did not reveal asymmetry in the other items, indicating a reliable analysis. The Egger test was calculated as $2.79(\mathrm{p}=0.17), 2.35(\mathrm{p}=0.35)$, $1.44(\mathrm{p}=0.40), 9.25(\mathrm{p}=0.19),-4.54(\mathrm{p}=0.04), 1.93(\mathrm{p}$ $=0.77),-8.18(\mathrm{p}=0.18)$, and $-1.26(\mathrm{p}=0.64)$ for ODI score, leg pain VAS score, back pain VAS score, ROM at the treated segment, ROM at the adjacent segment, EBL, surgery duration, and length of hospital stay, respectively. Results of Egger tests of comparisons were insignificant except for ROM at the adjacent segment; for this comparison of ROM at the adjacent segment, interpretation with caution is needed because of publication bias.

\section{Discussion}

In this meta-analysis of 7 studies involving 506 middleaged patients (mean age 50.3 years), treatment with dynamic stabilization using the Dynesys system, compared with conventional PLIF, resulted in similar clinical outcomes in approximately 2 years of follow-up, and better perioperative outcomes were seen in patients with spinal disease in the Dynesys group. Regarding the protective effect against adjacent-segment hypermobility, the Dynesys group was not substantially superior to the PLIF group.

\section{Evaluation of Possible Advantages of the Dynesys System}

The primary biomechanical goals of PDS devices are to preserve motion as much as possible while reducing spinal instability to achieve even load transmission. ${ }^{30}$ Many in vitro and biomechanical studies have shown that the Dynesys system can restrain the amount of flexibility through polyethylene-terephthalate cords and spacers. ${ }^{38}$ Jahng et al..$^{12}$ performed finite-element analysis and found that the ROM in patients treated with the Dynesys model declined by approximately $60 \%$ compared with those treated using an intact model. A number of cadaveric, in vivo, and clinical studies have provided conflicting results. $2,6,38,39$ Our meta-analysis shows that the ROM at the treated segment decreased by $42.0 \%$ in the Dynesys group and by $88.0 \%$ in the PLIF group. The Dynesys group maintained partial 
Surgery duration (minute)

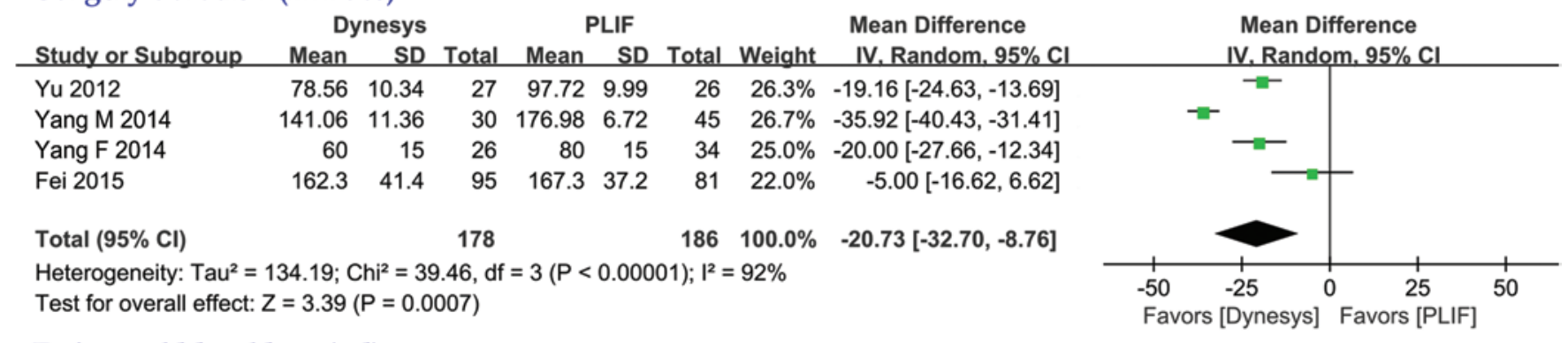

Estimated blood loss (ml)

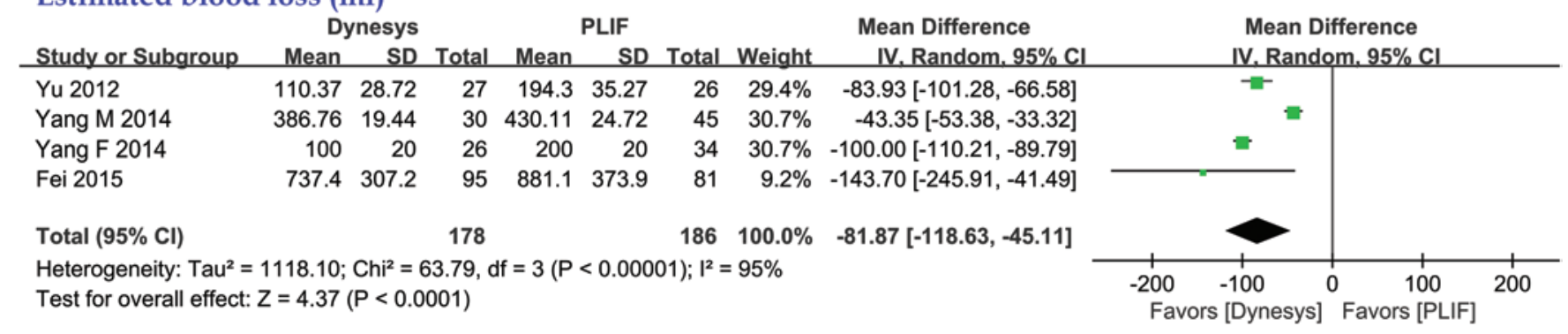

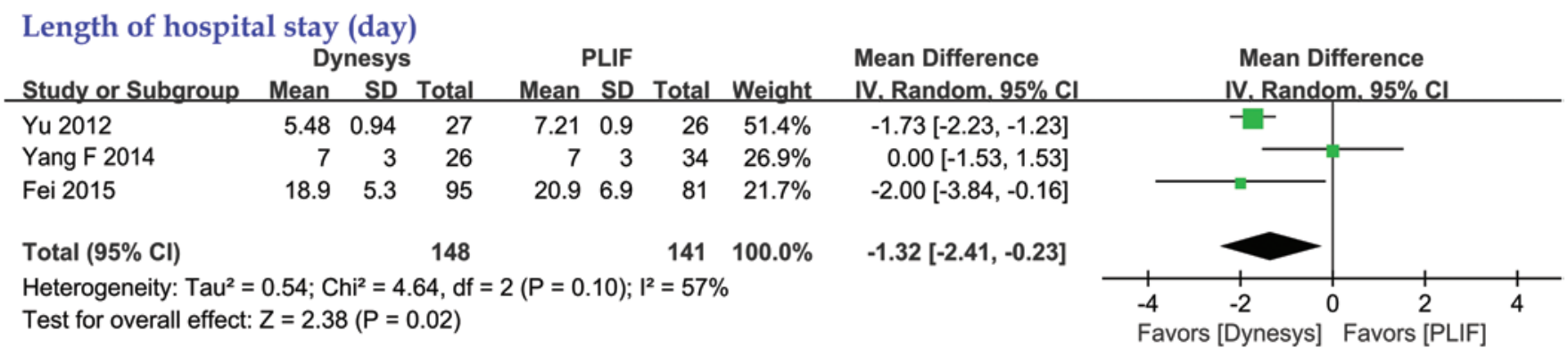

FIG. 2. Forest plots of surgery duration, EBL, and length of hospital stay. The Dynesys group had a mean of 20.73 fewer minutes of surgery time, $81.87 \mathrm{ml}$ less EBL, and 1.32 fewer days of hospital stay than the PLIF group. These differences between the 2 groups were statistically significant.

segmental motion and also achieved clinical and functional outcomes comparable with those of the PLIF group.

The chief advantage of dynamic stabilization may be its potential benefit in minimizing ASD. ${ }^{28}$ However, previous investigators have insisted that it is unclear whether such devices lead to better outcomes than those of traditional fusion, and it is unclear if they truly lead to a decrease in ASD. ${ }^{5}$ In regards to the protective effect against ASD, previous studies have had conflicting results. ${ }^{39}$ Our metaanalysis shows that the Dynesys group had no competitive advantage. Of 4 studies, 2 found that Dynesys provided an advantage, and 2 found that PLIF provided an advantage. The reasons for these contradictory results are unclear. One reason might be the difference in the pretension of the polyethylene-terephthalate cord by surgeons.,24,32 The Dynesys implantation guide recommends that a $300-\mathrm{N}$ preload be applied to the cords to distract the disc during the implantation procedure. ${ }^{29}$ The use of a $300-\mathrm{N}$ cord pretension causes a much higher stiffness at the implanted level than the intact lumbar spine. ${ }^{20}$ Because the effects of spacer length and cord tension on segmental flexibility and rigidity are still debated in the literature, many surgeons adjust the pretension below $300 \mathrm{~N}$ depending on the patient's condition. ${ }^{24,32}$
Other advantages of the Dynesys system are that the procedure to implant it is less invasive than PLIF and it results in earlier recovery. The proponents of dynamic stabilization claim that, compared with conventional rigid spinal fusion, dynamic stabilization results in less morbidity by being less invasive..$^{11,31}$ In short-term outcomes, our meta-analysis reveals that PDS provides a substantial advantage over PLIF in terms of recovery time. The Dynesys group showed a substantially reduced length of hospital stay, operative duration, and EBL. These results need to be interpreted with caution, because there are many confounding factors. Although the changes reached statistical significance, it is doubtful that a difference in blood loss of $80 \mathrm{ml}$ or hospitalization of 1.3 days is clinically relevant. Because there is no report of the minimum clinically important difference for them, the significance of these differences is unclear. There could have been some bias in the length-of-stay and time-of-discharge data. Some studies reported mean hospitalizations of more than 2 weeks and approaching 3 weeks. These lengths of stay are not normal; therefore, it is unclear whether there is truly a difference in hospitalization. One possible reason is that lengths of hospital stay may be affected by the medical environment and cultural aspects of medical practice in 


\begin{tabular}{|c|c|c|c|c|c|c|c|c|c|}
\hline \multirow[b]{2}{*}{ Study or Subgroup } & \multicolumn{3}{|c|}{ Dynesys } & \multicolumn{3}{|c|}{ PLIF } & \multirow[b]{2}{*}{ Weight } & \multirow{2}{*}{$\begin{array}{l}\text { Mean Difference } \\
\text { IV. Random. } 95 \% \mathrm{Cl}\end{array}$} & \multirow{2}{*}{$\begin{array}{l}\text { Mean Difference } \\
\text { IV. Random, } 95 \% \mathrm{Cl}\end{array}$} \\
\hline & Mean & SD & Total & Mean & SD & Total & & & \\
\hline Kaner $2010^{13}$ & 64.23 & 10.85 & 26 & 65.5 & 8.78 & 20 & $15.3 \%$ & $-1.27[-6.94,4.40]$ & \\
\hline Yu 2012 & 32.74 & 5.91 & 27 & 29.31 & 5.3 & 26 & $21.0 \%$ & $3.43[0.41,6.45]$ & \\
\hline Haddad 2013 & 17.31 & 17.8 & 32 & 28.19 & 18.22 & 32 & $9.9 \%$ & $-10.88[-19.71,-2.05]$ & \\
\hline Yang 2013 & 40.81 & 14.33 & 14 & 47.76 & 14.54 & 18 & $8.4 \%$ & $-6.95[-17.02,3.12]$ & \\
\hline Yang M 2014 & 39.3 & 4.49 & 30 & 33.75 & 4.96 & 45 & $22.6 \%$ & $5.55[3.39,7.71]$ & - \\
\hline Fei 2015 & 31.2 & 6.92 & 95 & 31.2 & 7.25 & 81 & $22.7 \%$ & $0.00[-2.10,2.10]$ & \\
\hline Total $(95 \% \mathrm{Cl})$ & & & 224 & & & 222 & $100.0 \%$ & $0.12[-3.48,3.72]$ & \\
\hline \multicolumn{9}{|c|}{$\begin{array}{l}\text { Heterogeneity: } \mathrm{Tau}^{2}=13.67 ; \mathrm{Ch}^{2}=27.09, \mathrm{df}=5(P<0.0001) ; \mathrm{I}^{2}=82 \% \\
\text { Test for overall effect: } Z=0.06(P=0.95)\end{array}$} & $\begin{array}{ccc}0 & 10 & 20 \\
& \text { Favors [Dynesys] }\end{array}$ \\
\hline
\end{tabular}

\section{Back pain VAS}

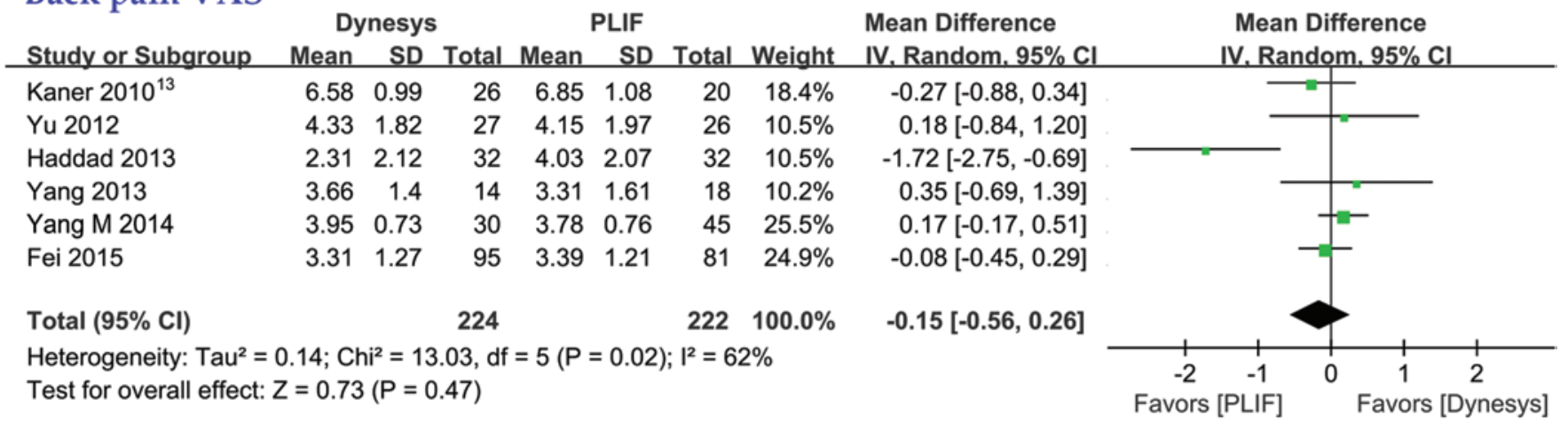

\section{Leg pain VAS}

\begin{tabular}{|c|c|c|c|c|c|c|c|c|c|c|}
\hline \multirow[b]{2}{*}{ Study or Subgroup } & \multicolumn{2}{|c|}{ Dynesys } & \multicolumn{3}{|c|}{ PLIF } & \multirow[b]{2}{*}{ Total } & \multirow[b]{2}{*}{ Weight } & \multirow{2}{*}{$\begin{array}{l}\text { Mean Difference } \\
\text { IV. Random, } 95 \% \mathrm{Cl}\end{array}$} & \multirow{2}{*}{$\begin{array}{c}\text { Mean Difference } \\
\text { IV. Random. } 95 \% \mathrm{Cl}\end{array}$} & \\
\hline & Mean & SD & Total & Mean & SD & & & & & \\
\hline Yu 2012 & 5.37 & 1.22 & 27 & 5.08 & 0.97 & 26 & $22.8 \%$ & $0.29[-0.30,0.88]$ & & \\
\hline Haddad 2013 & 2.53 & 2.08 & 32 & 3.88 & 2.24 & 32 & $10.8 \%$ & $-1.35[-2.41,-0.29]$ & & \\
\hline Yang M 2014 & 3.74 & 0.7 & 30 & 3.7 & 0.66 & 45 & $35.6 \%$ & $0.04[-0.28,0.36]$ & & \\
\hline Fei 2015 & 4.49 & 1.36 & 95 & 4.52 & 1.41 & 81 & $30.8 \%$ & $-0.03[-0.44,0.38]$ & & \\
\hline Total $(95 \% \mathrm{Cl})$ & & & 184 & & & 184 & $100.0 \%$ & $-0.07[-0.47,0.32]$ & & \\
\hline $\begin{array}{l}\text { Heterogeneity: } \mathrm{Tau}^{2} \\
\text { Test for overall effect }\end{array}$ & $\begin{array}{l}.09 ; \mathrm{Cr} \\
=0.37\end{array}$ & $\begin{array}{l}i^{2}=7 . \\
(P=C\end{array}$ & $\begin{array}{l}\text { 24, df }= \\
.71)\end{array}$ & $3(P=$ & 0.06 ); & $1^{2}=59$ & & & $\begin{array}{cc}-2 & -1 \\
\text { Favors [PLIF] }\end{array}$ & $\begin{array}{l}2 \\
\text { nesys] }\end{array}$ \\
\hline
\end{tabular}

FIG. 3. Forest plots of functional and clinical outcomes. The Dynesys and PLIF groups improved substantially in ODI and back and leg pain VAS scores after surgical treatment. There were no significant differences between the 2 groups, although the PLIF group had a better VAS score decrease than the Dynesys group.

specific regions. However, almost all the studies indicated that Dynesys provided an advantage. Although we did not compare items, it is obvious that there were no complications with bone graft material or the process of intradiscal space preparation in the Dynesys group, because PDS with the Dynesys did not necessitate fusion.

\section{Evaluation of Possible Disadvantages of the Dynesys System}

One major argument against the PDS systems including Dynesys is the possibility of screw loosening. ${ }^{27}$ Survival against fatigue failure is the biggest challenge for PDS devices because of the need for continued motion for an indefinite period. ${ }^{30}$ Demands for durability and mechanical strength of the PDS implant are higher than those required for fusion. ${ }^{16}$ Our meta-analysis shows that radiographically revealed and symptomatic screw loosen- ing in the Dynesys group was observed at rates of $2.54 \%$ and $0.42 \%$, respectively, which are not different than those of the PLIF group. Both the PDS and the PLIF groups had similar screw-loosening rates. The similarity of the screwloosening rates in the 2 groups could be explained by unintended fusion in the PDS group, but the ROM data did not bear that out. Previous single-treatment-arm studies of the Dynesys system also found radiographic evidence of screw loosening in $1.2 \%-19.8 \%$ of patients and $2.8 \%-$ $4.7 \%$ of screws, and clinical deterioration resulting from the loosening of screws and necessitating revision surgery occurred in $0 \%-1.2 \%$ of patients. ${ }^{16,17,26,30,31,34}$ Therefore, the risk of screw loosening and revision surgery after PDS using Dynesys seems to be considerably low.

Another argument against the Dynesys system is its poor outcomes compared with those of fusion in patients with spondylolisthesis and concomitant spinal stenosis. ${ }^{4}$ 


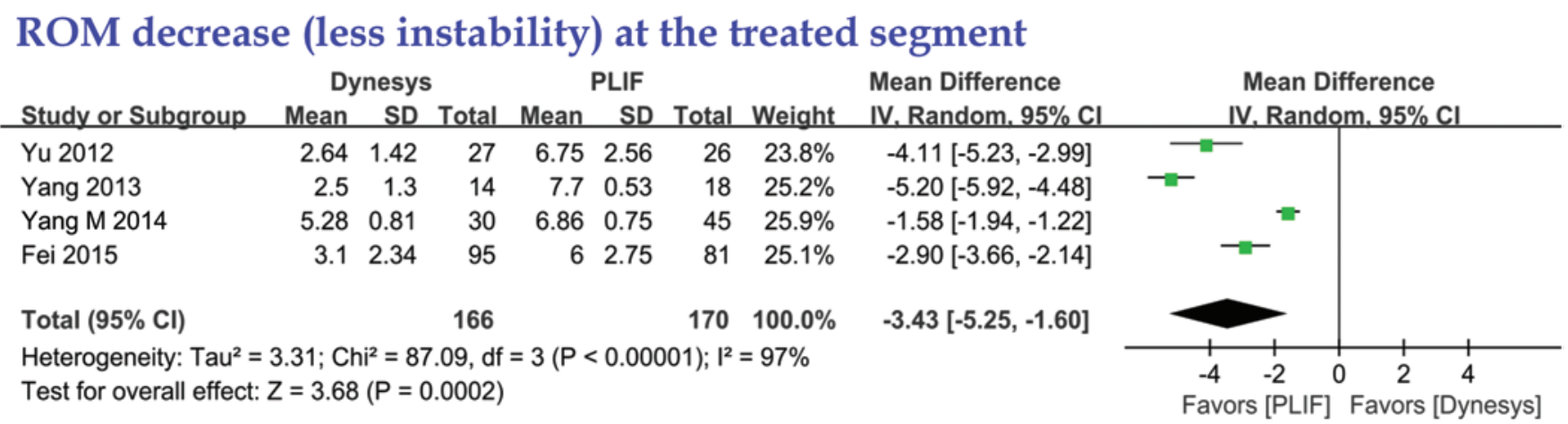

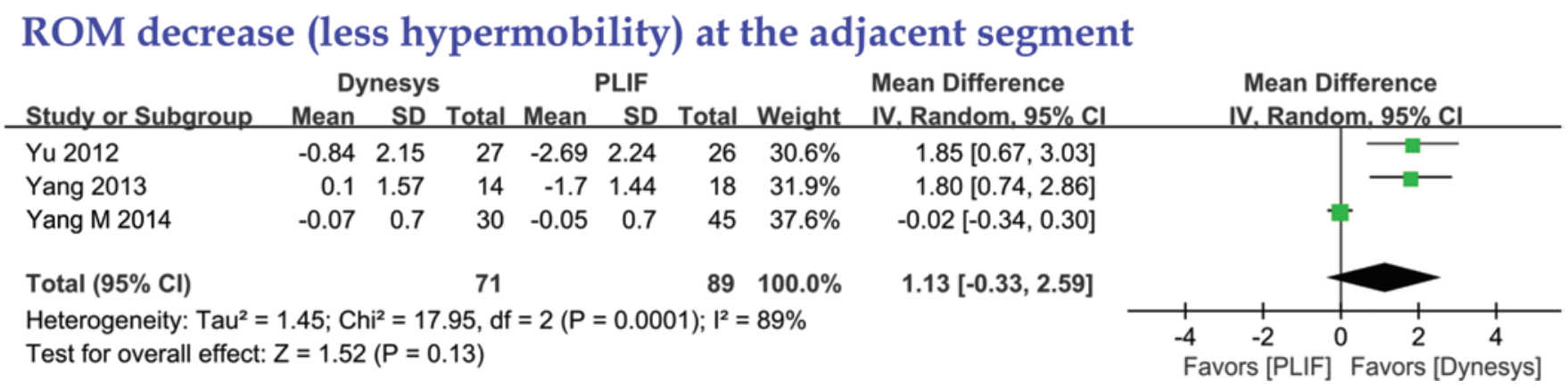

FIG. 4. Forest plots of ROM changes at the treated and adjacent segments. At the treated segment, ROM decreased substantially in both groups. The PLIF group showed significant ROM reduction compared with that of the Dynesys group. At the adjacent segment, both groups showed slightly increased ROM. There were no significant differences between the 2 groups, although the Dynesys group experienced less hypermobility than the PLIF group.

Seven studies in this analysis included patients with spondylolisthesis (Grade I). Although the rates of spondylolisthesis were $49 \%$ and $100 \%$ in the reports by Kaner et al. ${ }^{13}$ and $\mathrm{Yu}$ et al., ${ }^{39}$ the Dynesys groups in these studies had similar clinical and radiological outcomes that were not inferior to those of the PLIF groups. Previous reports also found that Dynesys provides similar radiographic stability and clinical effects regardless of preoperative spondylolisthesis. ${ }^{7}$ These previous studies usually dealt with Grade I spondylolisthesis and achieved results comparable to those that dealt with no spondylolisthesis., ${ }^{7,819}$ However, some investigators included Grade II spondylolisthesis and found favorable outcomes. ${ }^{14,26}$ Additional large studies are needed to clarify this issue.

\section{Limitations}

This study has some important limitations that must be considered when interpreting the results. Four of 7 studies were performed in China. The results have to be interpreted with caution because of uneven regional distribution. The number of treated segments varied from 1 to 3 . Moreover, 1 article did not describe the number of segments. ${ }^{11}$ Although studies of scoliosis surgery were excluded, this variance in the surgery can affect clinical outcomes. However, a previous study found that clinical improvements were not different between single and multilevel dynamic stabilization. ${ }^{15}$ ROM changes at the adjacent segment can be affected by various treated segments. Two of 4 included reports that described ROM at the adjacent segment did not explain whether the adjacent segment included the cranial or caudal segment of the treated level. ${ }^{36,38}$ We asked the authors to clarify the meaning of "adjacent segment," and only 1 replied that it was a "compound value" of both the cranial and caudal segments. ${ }^{38}$ Because we could not know the clear calculation method or the way in which the authors dealt with having no caudal adjacent segment in cases of L5-S1 surgery, the data were regarded as concerning the cranial adjacent segment. During Dynesys implantation, surgeons have to decide on the length of spacer and cord pretension, which are not required in PLIF surgery. Their values are debatable and can vary according to surgeon experiences and the patient's condition. We did not consider the differences between the surgical procedures in our meta-analysis. One study described results as change percentages (not means and standard deviations). ${ }^{39}$ Based on the baseline data (described as means and standard deviations) and change percentages, we calculated mean differences and standard deviations, as described previously., ${ }^{3,22,23}$ The calculated values were not exact but instead were estimates.

\section{Conclusions}

Both PDS and PLIF provide similar clinical outcomes in relatively young patients (in their 5th to 6th decade of life) who undergo surgery for degenerative disc disease or spinal stenosis with or without spondylolisthesis. The Dynesys system reduces instability at the treated segment; the level of ASD prevention by Dynesys is unclear. Fusion 

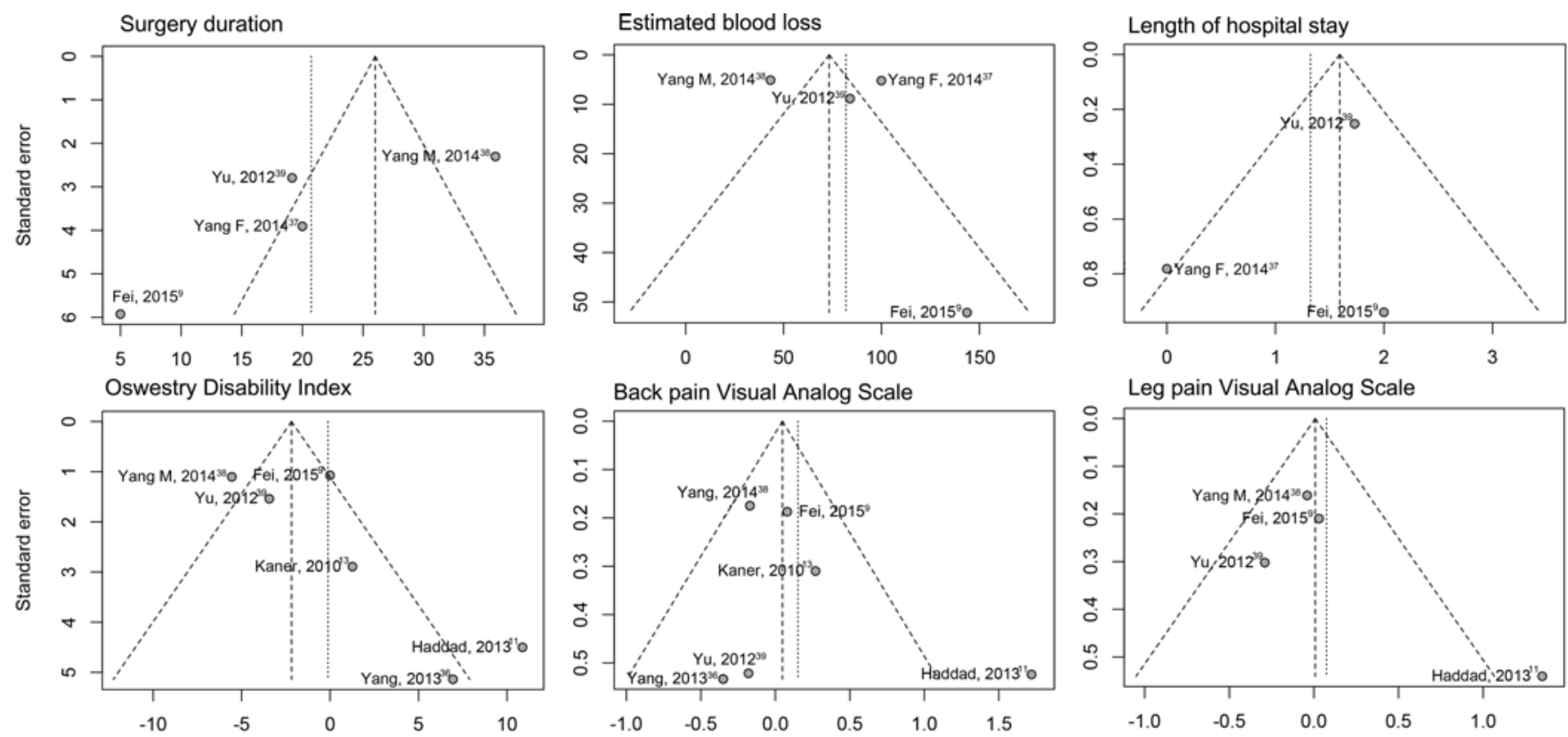

Back pain Visual Analog Scale
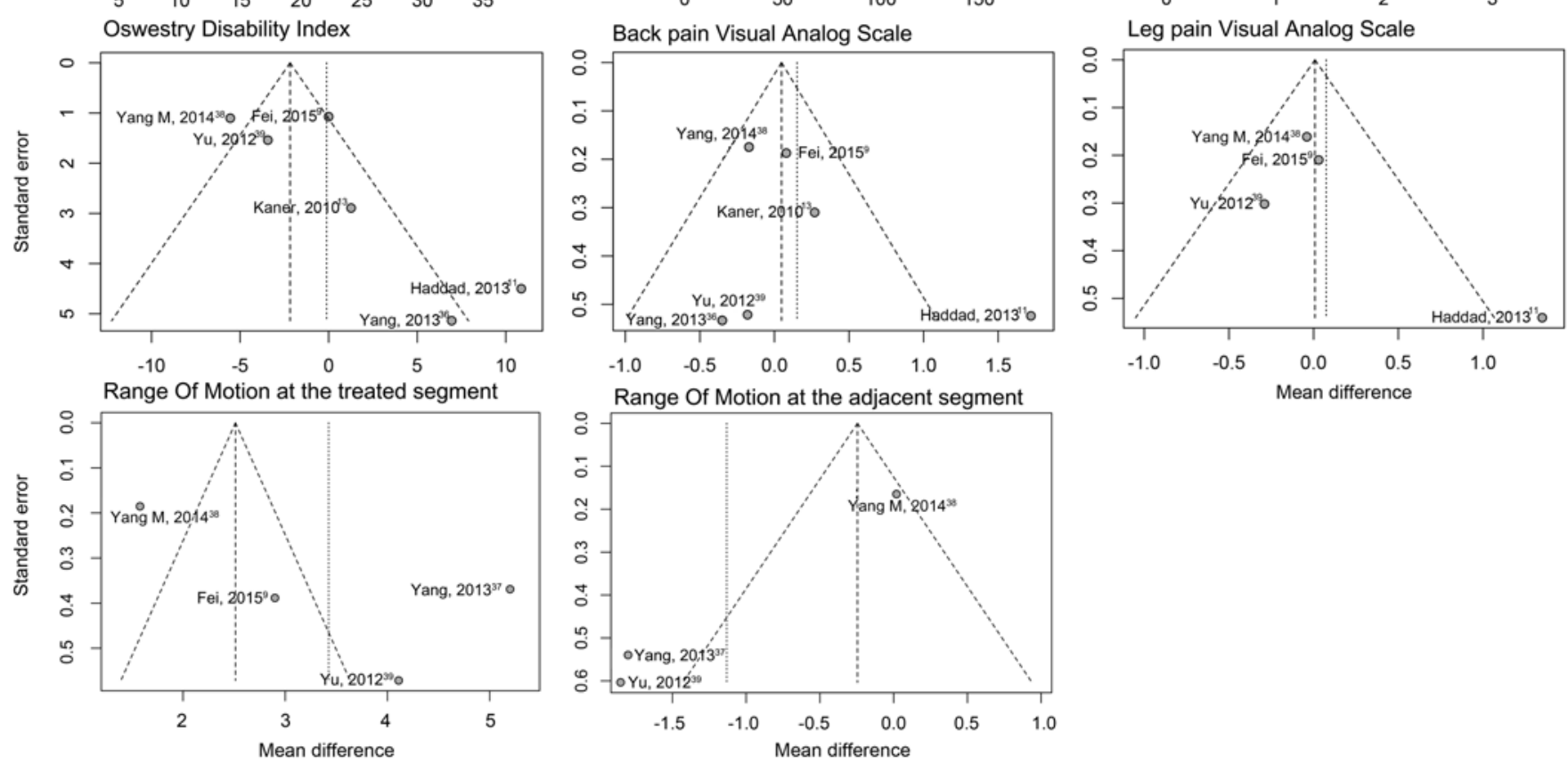

FIG. 5. Funnel plots of all items. All items show relatively symmetric plots except ROM. The asymmetry of the ROM data may have been caused by publication bias.

still remains the method of choice for advanced degeneration and gross instability. However, degenerative spinal disease with or without Grade I spondylolisthesis, particularly in patients who require a quicker recovery after surgery, will likely constitute the main indication for PDS using the Dynesys system.

\section{References}

1. Anand N, Baron EM: Role of Dynesys as pedicle-based nonfusion stabilization for degenerative disc disorders. Adv Orthop 2012:218385, 2012

2. Beastall J, Karadimas E, Siddiqui M, Nicol M, Hughes J, Smith F, et al: The Dynesys lumbar spinal stabilization system: a preliminary report on positional magnetic resonance imaging findings. Spine (Phila Pa 1976) 32:685-690, 2007

3. Becker BJ: Synthesizing standardized mean-change measures. Br J Math Stat Psychol 41:257-278, 1988

4. Cakir B, Carazzo C, Schmidt R, Mattes T, Reichel H, Käfer W: Adjacent segment mobility after rigid and semirigid instrumentation of the lumbar spine. Spine (Phila Pa 1976) 34:1287-1291, 2009

5. Chou D, Lau D, Skelly A, Ecker E: Dynamic stabilization versus fusion for treatment of degenerative spine conditions. Evid Based Spine Care J 2:33-42, 2011
6. Delank KS, Gercek E, Kuhn S, Hartmann F, Hely H, Röllinghoff M, et al: How does spinal canal decompression and dorsal stabilization affect segmental mobility? A biomechanical study. Arch Orthop Trauma Surg 130:285-292, 2010

7. Fay LY, Wu JC, Tsai TY, Tu TH, Wu CL, Huang WC, et al: Intervertebral disc rehydration after lumbar dynamic stabilization: magnetic resonance image evaluation with a mean followup of four years. Adv Orthop 2013:437570, 2013

8. Fayyazi AH, Ordway NR, Park SA, Fredrickson BE, Yonemura K, Yuan HA: Radiostereometric analysis of postoperative motion after application of Dynesys dynamic posterior stabilization system for treatment of degenerative spondylolisthesis. J Spinal Disord Tech 23:236-241, 2010

9. Fei H, Xu J, Wang S, Xie Y, Ji F, Xu Y: Comparison between posterior dynamic stabilization and posterior lumbar interbody fusion in the treatment of degenerative disc disease: a prospective cohort study. J Orthop Surg 10:87, 2015

10. Grob D, Benini A, Junge A, Mannion AF: Clinical experience with the Dynesys semirigid fixation system for the lumbar spine: surgical and patient-oriented outcome in 50 cases after an average of 2 years. Spine (Phila Pa 1976) 30:324-331, 2005

11. Haddad B, Makki D, Konan S, Park D, Khan W, Okafor B: Dynesys dynamic stabilization: less good outcome than lumbar fusion at 4-year follow-up. Acta Orthop Belg 79:97-103, 2013 
12. Jahng TA, Kim YE, Moon KY: Comparison of the biomechanical effect of pedicle-based dynamic stabilization: a study using finite element analysis. Spine J 13:85-94, 2013

13. Kaner T, Dalbayrak S, Oktenoglu T, Sasani M, Aydin AL, Ozer AF: Comparison of posterior dynamic and posterior rigid transpedicular stabilization with fusion to treat degenerative spondylolisthesis. Orthopedics 33:309, 2010

14. Kaner T, Sasani M, Oktenoglu T, Aydin AL, Ozer AF: Clinical outcomes of degenerative lumbar spinal stenosis treated with lumbar decompression and the Cosmic "semi-rigid" posterior system. SAS J 4:99-106, 2010

15. Kim CH, Chung CK, Jahng TA: Comparisons of outcomes after single or multilevel dynamic stabilization: effects on adjacent segment. J Spinal Disord Tech 24:60-67, 2011

16. Ko CC, Tsai HW, Huang WC, Wu JC, Chen YC, Shih YH, et al: Screw loosening in the Dynesys stabilization system: radiographic evidence and effect on outcomes. Neurosurg Focus 28(6):E10, 2010

17. Lee SE, Jahng TA, Kim HJ: Decompression and nonfusion dynamic stabilization for spinal stenosis with degenerative lumbar scoliosis: Clinical article. J Neurosurg Spine 21:585-594, 2014

18. Lee SE, Park SB, Jahng TA, Chung CK, Kim HJ: Clinical experience of the dynamic stabilization system for the degenerative spine disease. J Korean Neurosurg Soc 43:221-226, 2008

19. Lee SH, Lee JH, Hong SW, Chung SE, Yoo SH, Lee HY: Spinopelvic alignment after interspinous soft stabilization with a tension band system in grade 1 degenerative lumbar spondylolisthesis. Spine (Phila Pa 1976) 35:E691-E701, 2010

20. Liu CL, Zhong ZC, Hsu HW, Shih SL, Wang ST, Hung C, et al: Effect of the cord pretension of the Dynesys dynamic stabilisation system on the biomechanics of the lumbar spine: a finite element analysis. Eur Spine J 20:1850-1858, 2011

21. Moon KY, Lee SE, Kim KJ, Hyun SJ, Kim HJ, Jahng TA: Back muscle changes after pedicle based dynamic stabilization. J Korean Neurosurg Soc 53:174-179, 2013

22. Morris SB: Distribution of the standardized mean change effect size for meta-analysis on repeated measures. Br J Math Stat Psychol 53:17-29, 2000

23. Morris SB, DeShon RP: Combining effect size estimates in meta-analysis with repeated measures and independentgroups designs. Psychol Methods 7:105-125, 2002

24. Niosi CA, Zhu QA, Wilson DC, Keynan O, Wilson DR, Oxland TR: Biomechanical characterization of the three-dimensional kinematic behaviour of the Dynesys dynamic stabilization system: an in vitro study. Eur Spine J 15:913-922, 2006

25. Nockels RP: Dynamic stabilization in the surgical management of painful lumbar spinal disorders. Spine (Phila Pa 1976) 30 (16 Suppl):S68-S72, 2005

26. Schaeren S, Broger I, Jeanneret B: Minimum four-year follow-up of spinal stenosis with degenerative spondylolisthesis treated with decompression and dynamic stabilization. Spine (Phila Pa 1976) 33:E636-E642, 2008

27. Schnake KJ, Schaeren S, Jeanneret B: Dynamic stabilization in addition to decompression for lumbar spinal stenosis with degenerative spondylolisthesis. Spine (Phila Pa 1976) 31:442-449, 2006

28. Schroeder GD, Murray MR, Hsu WK: A review of dynamic stabilization in the lumbar spine. Oper Tech Orthop 21:235-239, 2011

29. Schwarzenbach O, Berlemann U: [Dynamic posterior stabilization with the pedicle screw system DYNESYS ${ }^{\circledR}$.] Oper Orthop Traumatol 22:545-557, 2010 (Ger)
30. Sengupta DK, Herkowitz HN: Pedicle screw-based posterior dynamic stabilization: literature review. Adv Orthop 2012:424268, 2012

31. Stoll TM, Dubois G, Schwarzenbach O: The dynamic neutralization system for the spine: a multi-center study of a novel non-fusion system. Eur Spine J 11 (Suppl 2):S170-S178, 2002

32. Tsai TH, Kung SS, Huang TY, Hwang YF, Chang CH, Hwang SL: Posterior dynamic stabilization system (Dynesys) with interbody fusion for treating two-segment lumbar degenerative disc disease. Formosan J Surg 46:116-122, 2013

33. Welch WC, Cheng BC, Awad TE, Davis R, Maxwell JH, Delamarter R, et al: Clinical outcomes of the Dynesys dynamic neutralization system: 1-year preliminary results. Neurosurg Focus 22(1): E8, 2007

34. Wu JC, Huang WC, Tsai HW, Ko CC, Wu CL, Tu TH, et al: Pedicle screw loosening in dynamic stabilization: incidence, risk, and outcome in 126 patients. Neurosurg Focus 31(4):E9, 2011

35. Würgler-Hauri CC, Kalbarczyk A, Wiesli M, Landolt H, Fandino J: Dynamic neutralization of the lumbar spine after microsurgical decompression in acquired lumbar spinal stenosis and segmental instability. Spine (Phila Pa 1976) 33:E66-E72, 2008

36. Yang B, Jiang T: [Comparative study of dynamic neutralization system and posterior lumbar interbody fusion in treating lumbar degenerative disease.] Zhongguo Xiu Fu Chong Jian Wai Ke Za Zhi 27:140-144, 2013 (Chinese)

37. Yang F, He DW, Zhu XD, Li JF: [Dynesys dynamic fixation and lumbar spinal fusion for treatment of lumbar degenerative disease: a clinical comparative study.] Acad J Second Mil Med Univ 35:1145-1150, 2014 (Chinese)

38. Yang M, Li C, Chen Z, Bai Y, Li M: Short term outcome of posterior dynamic stabilization system in degenerative lumbar diseases. Indian J Orthop 48:574-581, 2014

39. Yu SW, Yang SC, Ma CH, Wu CH, Yen CY, Tu YK: Comparison of Dynesys posterior stabilization and posterior lumbar interbody fusion for spinal stenosis L4L5. Acta Orthop Belg 78:230-239, 2012

\section{Disclosures}

The authors report no conflict of interest concerning the materials or methods used in this study or the findings specified in this paper.

\section{Author Contributions}

Conception and design: $\mathrm{CH}$ Lee. Acquisition of data: $\mathrm{CH}$ Lee, Hyun, SE Lee. Analysis and interpretation of data: $\mathrm{CH}$ Lee, $\mathrm{CH}$ Kim, Park. Drafting the article: Jahng, CH Lee. Critically revising the article: Jahng, CH Lee, Hyun, CH Kim, KJ Kim, HJ Kim. Reviewed submitted version of manuscript: Jahng, CH Lee, Hyun, CH Kim, Park, KJ Kim, Chung, SE Lee. Approved the final version of the manuscript on behalf of all authors: Jahng. Statistical analysis: CH Lee. Study supervision: Jahng, Park, KJ Kim, Chung, HJ Kim.

\section{Correspondence}

Tae-Ahn Jahng, Department of Neurosurgery, Spine Center, Seoul National University Bundang Hospital, Seoul National University College of Medicine, 300 Gumi-dong, Bundang-gu, Seongnam, Gyeonggi 463-707, Republic of Korea. email: taj@ snu.ac.kr. 